\title{
Исследование с помощью вызванных потенциалов функциональной эквивалентности механизмов восприятия и генерации эмоций
}

\author{
Дарья С. Алексеева*, Павел Н. Ермаков, Виталий В. Бабенко, Денис В. Явна \\ Южный федеральный университет, г. Ростов-на-Дону, Российская Федерация \\ *E-mail: alexeeva_ds@mail.ru
}

\begin{abstract}
Аннотация
Введение. Отмечается, что гипотеза функциональной эквивалентности находит полтвержление во многих фрункциональных системах мозга. ОАнако этот вопрос остается открытым в отношении механизмов, участвующих в восприятии и воспроизведении эмоций. Ставится цель определить и сопоставить Аинамику активности мозговых структур при восприятии и генерации эмоций. Новизна исследования определяется тем, что впервые сравнивается Аинамика вызванной активности при восприятии и генерации эмоций у одних и тех же испытуемых в условиях оАного эксперимента.

Методы. Испытуемым преАъявлялись фоотографиии Аиц с разным эмоциональным выражением и объекты, вызывающие разные эмоциональные реакции. Испытуемые Аолжны были сообщить, какую эмоцию выражает преАьявляемое ^ицо и какую эмоцию вызывает у них показываемый объект. Ответы служили основой А^я Аальнейшего группирования и усреАнения оррагментов электроэнцефралограммы с целью вылеления вызванных потенциалов (на нейтральные, позитивные и негативные лица и объекты). Электроэнцефалограмма регистрировалась в 128 отвеАениях, что позволило определить траектории орокусов максимальной активности с помощью SLORETA. Сравнива^ись реакции на Аица и объекты оАинаковой эмоциона^ьной валентности.
\end{abstract}

Результаты. Впервые в рамках олного эксперимента регистрировались и сравнивались реакции на зрительные стимулы, отображающие и вызывающие эмоции. Различия межау вызванными потенциалами на мица с разной эмоциональной экспрессией и объекты с разной эмоциональной валентностью обнаружены на во^не N170. Анализ траекторий фокусов максимальной активности при развитии реакций на мица и объекты не выявил их пересечения.

ОбсужАение результатов. Указывается, что экспериментальные залачи были организованы таким образом, чтобы максимально развести Аинамику сравниваемых реакций. В этих условиях совпаление процессов возможно только на опреАеленном этапе в случае существования механизма «отзеркаливания». Анализ полученных результатов показал отсутствие пересечения сравниваемых процессов. 
Заключение. Полученные результаты не выявили признаков фрункциональной эквивалентности механизмов, распознающих и генерирующих эмоции.

\title{
КАючевые слова
}

^ица, восприятие эмоций, отзеркаливание, объекты, эмоциона^ьная реакция, генерация эмоций, эмоциональная экспрессия, эмоциональная валентность, вызванные потенциалы, фокусы максимальной активности

\section{Основные положения}

- вызванные потенциалы на ^ица с определенной эмоциональной экспрессией и объекты с той же эмоциональной валентностью существенно различаются; - траектории фокусов максимальной корковой активности в реакциях на ^ица и объекты с оАной и той же эмоциональной валентностью не пересекаются; - не обнаружено перекрытия механизмов восприятия и генерации эмоций.

\section{Благодарности}

Исследование выполнено при финансовой поддержке Минобрнауки РФ (проект № 25/3336.2017/ПЧ).

\section{Для цитирования}

Алексеева Д.С., Ермаков П.Н., Бабенко В.В., Явна Д.В. Исследование с помощью вызванных потенциалов функциональной эквивалентности механизмов восприятия и генерации эмоций // Российский психологический журнал. - 2017. - Т. 14, № 4. - С. 97-120.

\section{Studying the Functional Equivalence of Mechanisms for Emotion Perception and Emotion Generation by Means of Evoked Potentials}

\author{
Dar'ya S. Alekseeva*, Pavel N. Ermakov, Vitalii V. Babenko, Denis V. Yavna \\ Southern Federal University, Rostov-on-Don, Russian Federation \\ *Correspondence author. E-mail: alexeeva_ds@mail.ru
}

\begin{abstract}
Introduction. Many functional systems of the brain confirm the hypothesis of functional equivalence. However, the mechanisms involved in emotion perception and reproduction still remain an open question. This study attempts to define and compare
\end{abstract}




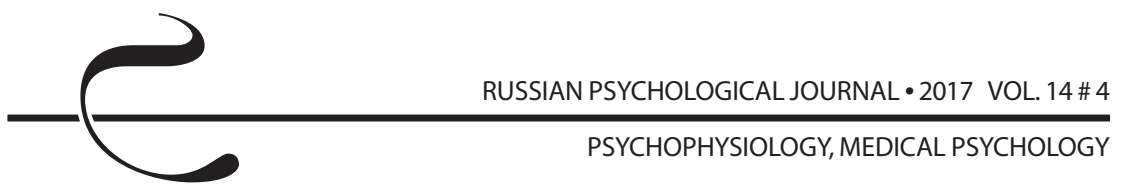

the dynamics of the activity of brain structures when perceiving and generating emotions. The novelty of the research lies in comparing the dynamics of evoked activity when the same respondents perceive and generate emotions within a single experiment.

Methods. The respondents were shown photographs of various facial expressions and also objects generating various emotions. The respondents had to detect emotions from photographs of facial expressions, and also recognize emotions which the objects generated. The answers made it possible to group and average EEG fragments to distinguish evoked potentials (neutral, positive, and negative faces and objects). EEG was recorded from 128 derivations, which allowed determining the trajectories of the foci of maximal activity using sLORETA. The responses to faces and objects of the same emotional valence were compared.

Results. For the first time the responses to the visual stimuli expressing and generating emotion were recorded and compared within a single experiment. The N170 wave activity displayed the differences between evoked responses to different facial expressions and objects with different emotional valence. The analysis of the trajectories of the foci of maximal activity when developing reactions to faces and objects showed no crossing.

Discussion. The experiment should have parted the dynamics of the compared responses. Assuming the existence of a mirror mechanism, the coincidence of the processes is possible only at a certain stage in this case. The analysis of the results demonstrated no crossing for the compared processes.

Conclusion. The findings showed no signs of the functional equivalence of the mechanisms for emotion recognition and emotion generation.

\section{Keywords}

faces, emotion perception, mirroring, objects, emotional reaction, emotion generation, emotional expression, emotional valence, evoked potentials, foci of maximal activity

\section{Highlights}

- Evoked responses to various facial expressions and objects with the same emotional valence differ significantly.

- When reacting to faces and objects with the same emotional valence the trajectories of the foci of maximal cortical activity do not cross.

- The mechanisms for emotion perception and emotion generation do not overlap.

\section{Acknowledgments}

The study was supported by the Ministry of Education and Science of the Russian Federation (project no. 25/3336.2017/ПЧ). 


\section{For citation}

Alekseeva D. S., Ermakov P.N., Babenko V.V., Yavna D.V. Studying the Functional Equivalence of Mechanisms for Emotion Perception and Emotion Generation by Means of Evoked Potentials. Rossiiskii psikhologicheskii zhurnal - Russian Psychological Journal, 2017, V. 14, no. 4, pp. 97-120 (in Russian).

Original manuscript received 09.06.2017

\section{Введение}

Одна из замечательных способностей нашего мозга - способность имитировать ощущения, движения и другие виды опыта. Благодаря такому когнитивному моделированию мы имеем возможность представлять перцептивную информацию в нашем сознании и в отсутствие соответствующего сенсорного ввода.

Работы Р.Н. Шепарда и С. М. Косслина $[1,2]$ положили начало формированию представлений о сходстве форматов восприятия и воображения. Оказалось, что мысленное воспроизведение пространственных трансформаций объектов пропорционально времени их реальных трансформаций при наблюдении.

Позднее были обнаружены сходные эффекты при восприятии движения другого человека и при мысленном воспроизведении этого движения (напр., [3, 4]). Было показано, что двигательные образы приводят к активации тех же областей мозга, что и фактическое движение. Эти и прочие аналогичные результаты [5] интерпретировались авторами в качестве подтверждения гипотезы функциональной эквивалентности как одного из базовых принципов функциональной организации мозга человека.

Вполне естественно, что был поставлен и вопрос о том, распространяется ли принцип функциональной эквивалентности на механизмы восприятия и генерации эмоций. Действительно, система копирования чужих эмоций должна обеспечивать лучшее понимание собственных ощущений. Однако, с другой стороны, не менее важная задача - разделение репрезентаций своих эмоций и эмоций другого [6].

Таким образом, ответ на этот вопрос изначально не выглядел очевидным. И все же к началу 2000-х гг. сформировалось мнение, что восприятие эмоций и их генерация задействуют одни и те же механизмы (см. обзор [7]). Однако не все были согласны с этой точкой зрения, подчеркивая лишь частичное перекрытие механизмов восприятия и генерации эмоций [8]. Увеличение разрешающей способности томографов лишь укрепило эти сомнения. В итоге Т. Зингер и К. Ламм приходят к выводу, что активация мозга в процессе переживания и наблюдения эмоции пересекается, но не так сильно, как 
представлялось ранее [9]. А Ж. Десети, в свою очередь, констатирует, что на сегодня нет убедительных доказательств вовлечения зеркальных нейронов в восприятие эмоций [10].

Обратимся к работам, в которых говорится о сходстве рассматриваемых механизмов. Анализ этих исследований показывает, что результаты были получены главным образом в условиях, когда восприятие и генерация эмоций происходили у испытуемых практически одновременно. Например, наблюдатель видит яркие эмоции другого человека и сопереживает ему [11, 12]. Или же испытуемые должны наблюдать и воспроизводить эмоциональные выражения лиц (причем в отсутствие собственных эмоциональных переживаний) [13]. Как справедливо отмечают Б. Викер с соавторами, вполне возможно, что в этом случае в основе совпадения механизмов может лежать бессознательная имитация [14]. На это же указывают и недавние результаты А. Геллера с соавторами [15]. Но в любом случае речь здесь идет об одновременном развитии процессов восприятия и воспроизведения эмоций. И сложно представить, что в этих условиях можно было получить какой-либо иной результат, кроме совпадения механизмов.

Если же мы вспомним классическую работу Р. Н. Шепарда и Ж. Метцлер, в которой впервые была сформулирована гипотеза функциональной эквивалентности [1], то обратим внимание, что задачи восприятия и воспроизведения были разнесены во времени и исследовались независимо друг от друга.

Поскольку в работах с механизмами восприятия и генерации эмоций такого разделения сделано не было (в рамках одного исследования), мы предприняли попытку сопоставить результаты, полученные разными авторами, когда одни использовали задачу восприятия эмоциональных выражений лиц, в то время как другие демонстрировали сцены, способные вызвать эмоциональные переживания. Действительно, можно отметить существенное совпадение активируемых структур при восприятии эмоциональных лиц и эмоциональных сцен. Речь идет о миндалине, гиппокампе, префронтальной и зрительной коре, верхневисочной извилине, островке, передней поясной извилине... Такое сопоставление полезно, но, конечно, не отвечает на вопрос о функциональной эквивалентности в эмоциональной сфере. Ведь речь идет о результатах, полученных на разных испытуемых в разных экспериментальных условиях.

Еще одна проблема при сопоставлении реакций на лица и сцены состоит в том, что сцены - это группы объектов, которые часто (для усиления эмоциональности) включают людей. И именно выражение их лиц придает сцене ту или иную эмоциональную окраску. Другими словами, мы вновь сталкиваемся с перекрытием процессов восприятия и генерации эмоций. 
И вполне естественно, что в своем недавнем обзоре К. Ламм и Ж. Майданджич вынуждены констатировать, что вопрос о функциональной эквивалентности механизмов восприятия и генерации эмоций остается открытым [16].

Цель нашего исследования - определить и сопоставить динамику вызванной мозговой активности при восприятии и генерации эмоций.

Для достижения поставленной цели и получения принципиально новых результатов, позволяющих судить о совпадении или несовпадении механизмов оценки и генерации эмоций, следовало соблюсти несколько важных экспериментальных условий:

1. Мы осознанно сделали выбор в пользу использования вызванных потенциалов (128 отведений), поскольку, в отличие от томографии, они способны дать более точную информацию о динамике интересующего нас процесса.

2. В качестве стимулов, не выражающих, но вызывающих эмоции, мы использовали отдельные объекты (в отличие от традиционно используемых зрительных сцен).

3. Для классификации объектов по эмоциональной валентности мы применяли не априорное деление стимулов на группы (как это сделано в базах зрительных сцен), а ориентировались на оценку самих испытуемых. Например, чайник для большинства является нейтральным объектом. Однако у некоторых он может вызывать положительные эмоции, если связан с воспоминаниями о встречах с близкими людьми. Или, напротив, отрицательные эмоции, если он когда-то послужил причиной сильного ожога. Очевидно, что именно индивидуальный жизненный опыт формирует эмоциональную валентность объекта.

4. Мы регистрировали реакции на лица и объекты в одном эксперименте, что позволяло корректно сопоставлять динамику активности при восприятии и генерации эмоций.

5. Мы привлекли к экспериментам в качестве испытуемых представителей одного пола, поскольку эмоциональные реакции мужчин и женщин отличаются [17].

\section{Методы}

Испытуемые. В эксперименте на добровольной основе с соблюдением всех этических норм приняли участие 44 женщины в возрасте 18-20 лет, обладающие нормальным либо корригируемым до нормы зрением.

Стимулы. В эксперименте использовались два класса стимулов: чернобелые фотографии лиц и объектов.

Изображения лиц отбирались из следующих баз данных: Warsaw Set of Emotional Facial Expression Pictures (WSEFEP) [18], MMI Facial Expression 
Database [19] и Karolinska Directed Emotional Faces (KDEF) [20]. Было отобрано 175 моделей. При этом каждое лицо было представлено в трех вариантах: нейтральное, улыбающееся и грустное. Таким образом, всего в эксперименте использовалось 525 изображений лиц.

Было также подобрано 447 фотографий объектов, из которых 147 были условно маркированы нами как нейтральные, 154 - как способные вызвать у наблюдателя негативные эмоции и 146 - как способные вызвать позитивные эмоции.

Все изображения (и лица и объекты) были приведены к единому размеру. Для этого они вписывались в условную окружность диаметром 880 пикселей (около 14 угл. град.). Также они были выровнены по яркости и контрасту и помещены на равномерно серый фон, яркость которого равнялась средней яркости изображения $\left(30 \mathrm{kд} / \mathrm{m}^{2}\right)$.

На рисунке 1 представлены примеры стимульных изображений.

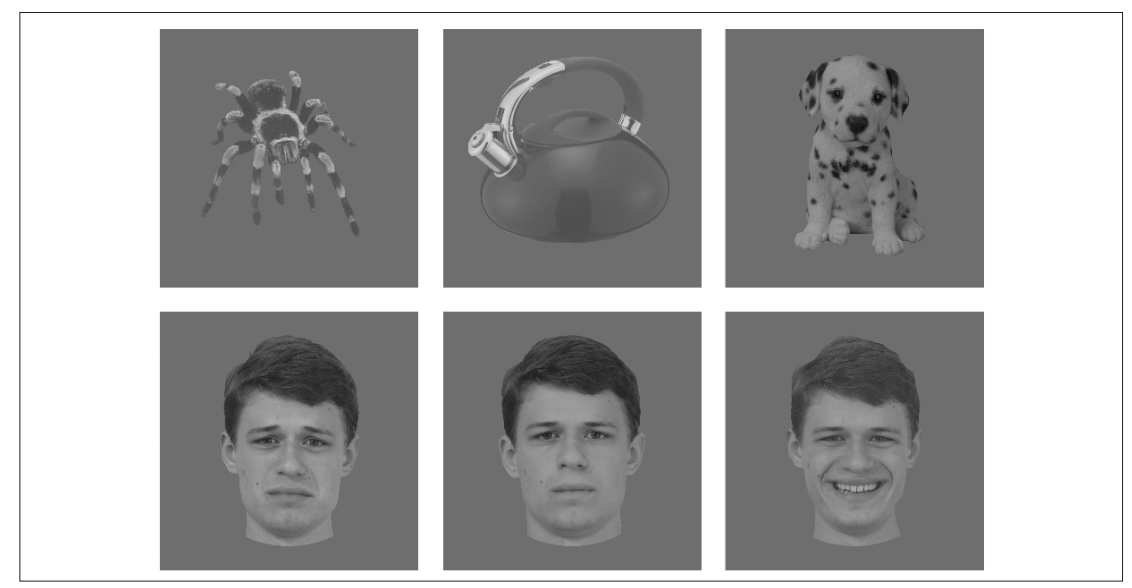

Рисунок 1. Примеры стимульных изображений

Примечание: верхний ряд - объекты с разной эмоциональной валентностью. Нижний ряд - лица с разным эмочиональным выражением.

Figure 1. Stimulus images

Note: the top row contains objects with different emotional valence. The bottom row represents various facial expressions.

Процедура. Эксперимент проводился в условиях искусственного освещения порядка 105 лк. Испытуемый располагался на расстоянии 90 см от монитора, на котором в течение 500 мс показывались подготовленные изображения. 
В одной части эксперимента стимулами служили лица, в другой - объекты. Последовательность предъявления изображений была случайной.

Когда в качестве стимулов выступали лица, задачей испытуемого было определить их выражение (радостное, грустное или нейтральное) и сообщить о своем решении нажатием соответствующей клавиши на компьютерной клавиатуре. Когда стимулами служили объекты, испытуемый должен был сообщить, какие эмоции вызывает у него предъявленный объект (позитивные, негативные, либо не вызывает никаких эмоций). Цифровые клавиши «1», «2» и «3» были промаркированы обозначениями «Negative», «Neutral» и «Positive». Введение ответа запускало демонстрацию следующего изображения после межстимульного интервала случайной длительности от 500 до 1500 мс.

Регистрация ЭЭГ. Регистрация ЭЭГ осуществлялась с помощью энцефалографа «Нейровизор 136» (ООО «Медицинские компьютерные системы», г. Москва, а. о. Зеленоград) в 128 монополярных отведениях с использованием шлема с комплектом съемных Ag-Cl электродов MCScap-AC128. Электроды располагались по системе «10-5». Оцифровывание ЭЭГ осуществлялось с частотой 1000 Гц.

Обработка. Конвертация меток событий (момент предъявления стимулов) выполнялась в программе EDFBrowser (разработчик - Teunis van Beelen). Для дальнейшей обработки использовались отрезки ЭЭГ, включающие 100 мс перед подачей стимула и 500 мс после начала предъявления. Анализ ограничивался первыми 500 мс, поскольку далее следовали колебания, связанные с моторным ответом испытуемого и активными глазными движениями.

Записи ЭЭГ сортировались в соответствии с ответами испытуемых относительно эмоциональной валентности стимулов. В результате для лиц и объектов было сформировано по 3 набора записей: реакции на нейтральные, позитивные и негативные лица, реакции на нейтральные, позитивные и негативные объекты. После выбора безартефактных записей они усреднялись в пределах каждого сформированного набора с целью выделения вызванных потенциалов (ВП). В результате для каждого испытуемого в каждом из 128 отведений было получено по 6 ВП: на нейтральные, позитивные и негативные лица; на нейтральные, позитивные и негативные объекты.

Для усреднения полученных ВП по всей выборке (grandaverage), построения карт распределения потенциалов и статистического сравнения реакций применялись средства пакета программ EEGLAB [21] для вычислительной среды Matlab. Локализация фокусов корковой активности определялась с помощью программы sLORETA [22].

Сопоставление ВП на лица и объекты с одинаковой эмоциональной валентностью осуществлялось путем помиллисекундного сравнения амплитуд с использованием критерия Стьюдента с поправкой Холма $(\mathrm{p}<0,01)$. 

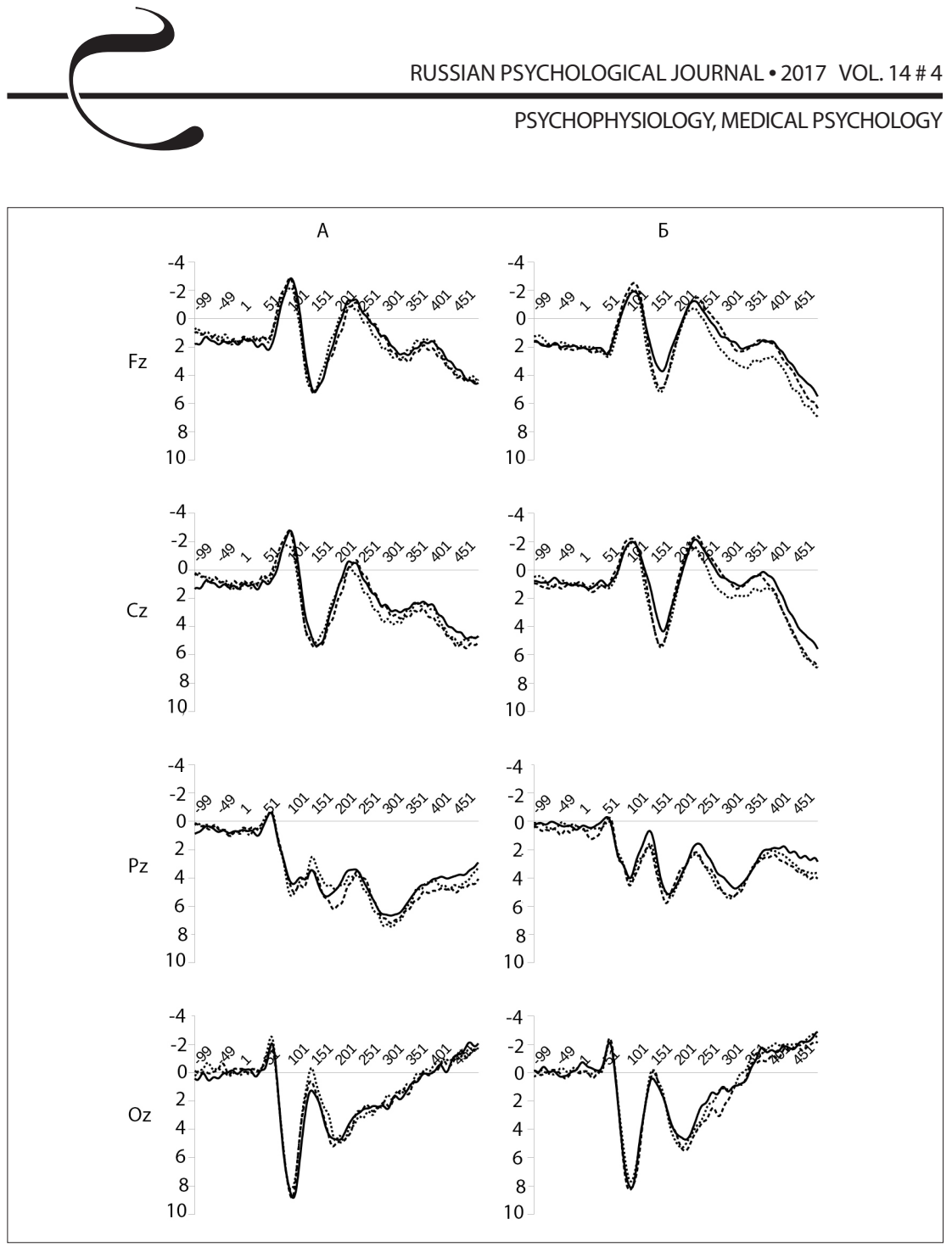

Рисунок 2. Усредненные ВП в лобном (Fz), центральном (Cz), теменном (Pz) и затылочном (Oz) отведениях в ответ на стимулы разных типов

Примечание: сплошной линией обозначены ВП на нейтральные стимулы, пунктиром - на негативные, точечным пунктиром - на позитивные. Ось абсцисс - время относительно предъявления стимула (мс). Ось ординат - амплитуда ВП (мкВ).

Figure 2. Averaged evoked responses to stimuli of different types recorded from frontal (Fz), central (Cz), parietal (Pz), and occipital (Oz) derivations

Note: The solid line represents evoked responses to neutral stimuli. The dashed line represents evoked responses to negative stimuli. The dotted line represents evoked responses to positive stimuli. The $x$-axis shows the time relative to the stimulus presentation (msec). The y-axis shows the evoked response amplitude ( $\mu \mathrm{V}$ ). 


\section{Результаты}

После усреднения ВП по 44 испытуемым мы получили в каждом из 128 отведений по 6 кривых, отражающих реакции мозга на предъявление нейтральных, позитивных и негативных лиц, а также нейтральных, позитивных и негативных объектов. На рисунке 2 представлены примеры усредненных ВП для каждого типа стимулов в лобном (Fz), центральном (Cz), теменном (Pz) и затылочном (Oz) отведениях. Рисунок иллюстрирует типичное инвертирование компонентов ВП на лобно-затылочной оси.

После получения усредненных ВП мы приступили к их сравнению в парах: нейтральное лицо - нейтральный объект, негативное лицо - негативный объект, позитивное лицо - позитивный объект. Помиллисекундное сравнение амплитуд ВП показало, что статистически значимые отличия возникают во множестве отведений и наблюдаются главным образом со 120-й по 170-ю мс. На рисунке 3 приведены топографические карты распределения потенциалов в указанном временном интервале с шагом 10 мс. Различия в сравниваемых картах (лица vs объекты одинакового эмоционального содержания) оценивались с помощью Т-критерия Стьюдента ( $<$ < 0,01). Множественность сравнений корректировалась поправкой Холма. Точками показаны локализации, в которых различия потенциалов статистически значимы. Видно, что значимые отличия сравниваемых реакций сосредоточены главным образом в лобно-центральных и височных отведениях.

На анализируемом временном интервале фокус позитивности смещается из затылочных областей в лобные. Для стимулов-лиц, выражающих негативную эмоцию, скорость этого смещения выше, чем для объектов, которые оценивались испытуемыми как негативные. В паре сравнения нейтральных лиц и объектов динамика реакций примерно одинакова. Ответы на объекты, воспринимаемые как позитивные, заметно опережают реакции на лица, выражающие положительную эмоцию. Значимые отличия в реакциях на эмоционально окрашенные стимулы возникают раньше, чем на нейтральные. Эти отличия нарастают быстрее при анализе изображений, оцениваемых как негативные.

Рисунок 4 позволяет лучше понять характер различий в реакциях, вызываемых лицами и объектами. На этом рисунке в качестве примера представлены кривые сравниваемых ВП в одном из отведений. Видно, что во всех трех парах сравнений отличия обусловлены существенным приростом позитивности в реакциях на объекты по сравнению с реакциями на лица. И хотя этот прирост охватывал все основные компоненты ВП, статистически значимыми они были лишь на коротком временном интервале, приходящемся на восходящую часть волны N170. Сами же ответы сильнее всего отличались в паре «нейтральное лицо - нейтральный объект». Во всех трех парах отличия 


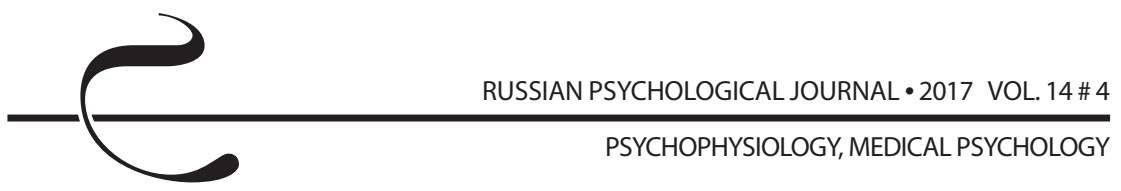

возникали примерно с одинаковой латентностью (125-130 мс), но завершались в разное время: быстрее всего для позитивных стимулов (133 мс), позднее всего - для нейтральных (152 мс).

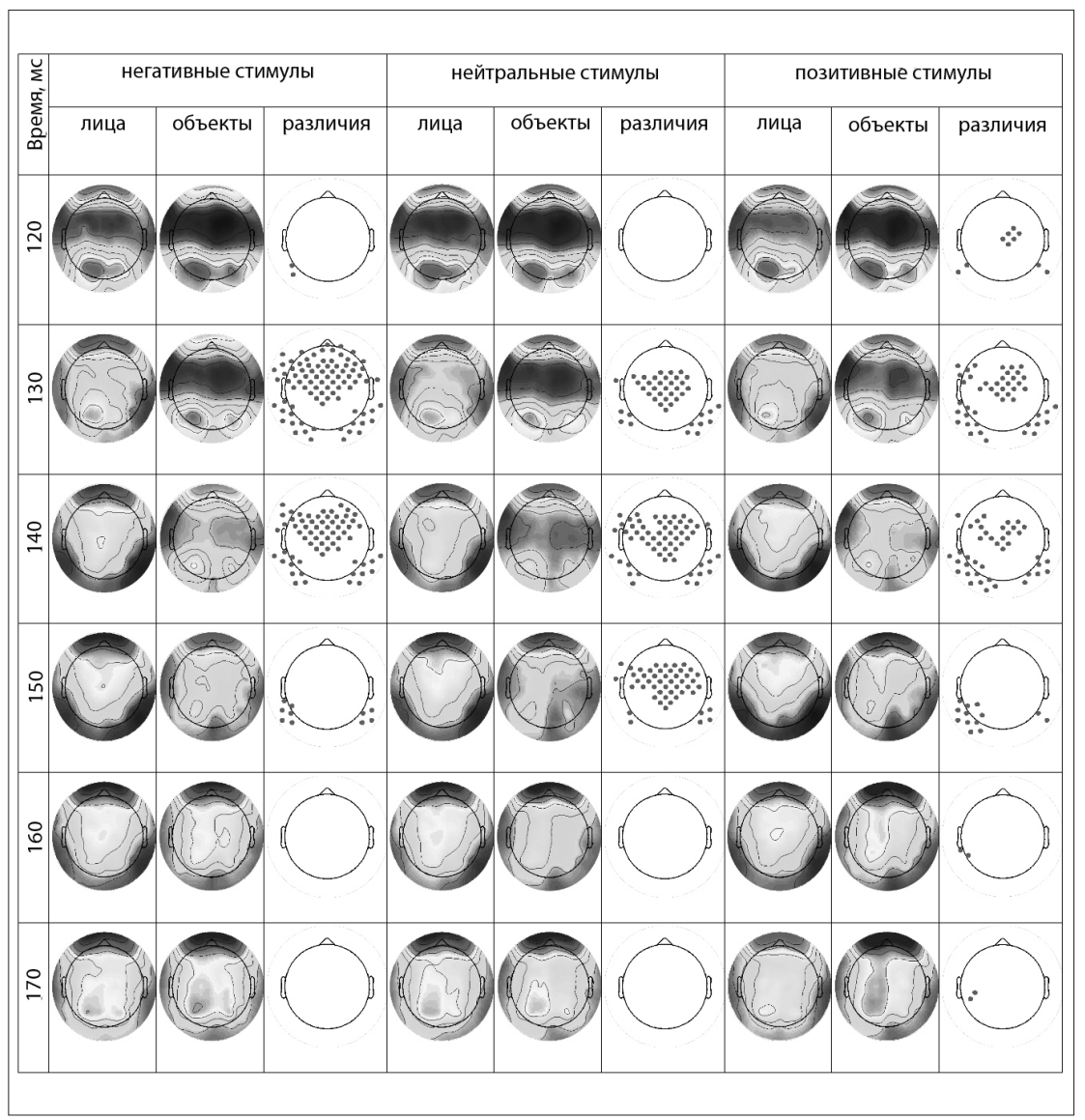

Рисунок 3. Карты потенциалов во временном интервале 120-170 мс (с шагом в 10 мс) от начала предьявления стимула

Примечание: красным иветом обозначена позитивность, синим - негативность. Точками указаны отведения, в которых отличия сравниваемых карт статистически значимы $(p<0,01)$.

Figure 3. Maps of potentials in the time interval between 120-170 msec (in $10 \mathrm{msec}$ increments) since the stimulus presentation

Note: Red color indicates positivity. Blue color indicates negativity. Dots indicate derivations where the compared differences are statistically significant $(p<0.01)$. 


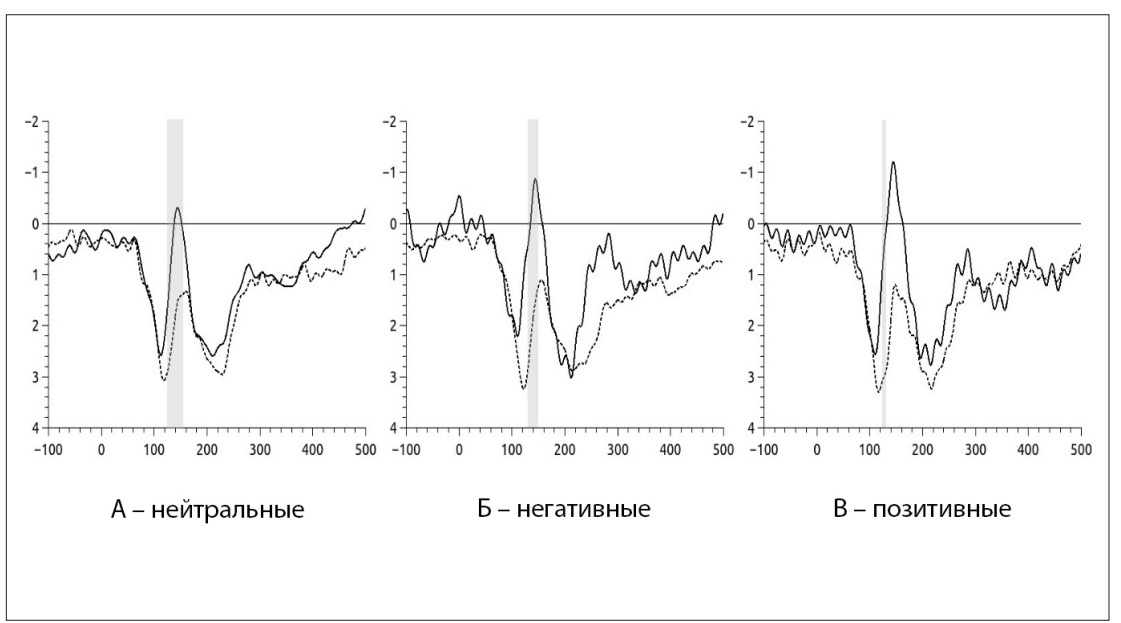

Рисунок 4. Усредненные ВП в отведении Трр9h на стимулы разной эмоциональной валентности

Примечание: сплошной линией обозначены ВП на лица, пунктирной - на объекты. Временные интервалы, в которых отмечены статистически значимые отличия (сравнение амплитуд по Т-критерию Стьюдента с поправкой Холма, $p<0,01)$, отмечены серым фоном. Значения Т-критерия, соответствующие максимальным различиям амплитуд в указанных временных диапазонах, составили для нейтральных стимулов 6,814, для негативных - 7,411, для позитивных - 7,996. По осям абсиисс указано время относительно подачи стимулов (мс), по осям ординат - амплитуды ВП (мкВ).

Figure 4. Averaged evoked responses to the stimuli of different emotional valence from the Tpp9h derivation

Полученные результаты указывают на то, что основные отличия сравниваемых ответов приходятся на компонент N170. Считается, что именно в этом компоненте отображаются процессы, связанные с обработкой лиц [23, 24], в том числе и реакции на эмоциональную экспрессию $[25,26]$. А значит, именно на этом временном интервале следует искать возможное «пересечение» реакций на лица и объекты, как отражение работы зеркальных нейронов, участвующих в отображении как чужих, так и собственных эмоций.

Чтобы определить динамику активности мозговых структур во время формирования волны N170, мы воспользовались методом SLORETA. Программа, реализующая этот метод, графически отображает распределение в коре текущей активности и позволяет с заданным временным шагом определять координаты максимальной активности с точностью \pm 5 мм [27]. Это дало нам возможность сравнить траектории фокусов активности во время развития реакций при предъявлении лиц (восприятие эмоций) и объектов (генерация 
эмоций). Именно пересечение сравниваемых траекторий может указывать на наличие механизмов «отзеркаливания». Результаты приведены в таблицах 1 и 2.

Таблица 1. Расстояние межАу фокусами активности при восприятии лиц, выражающих отрицательные эмоции, и при восприятии объектов, вызывающих отрицательные эмоции

Table 1. Distance between the foci of activity when perceiving negative facial expressions and also objects generating negative emotions

\begin{tabular}{|c|c|c|c|}
\hline \multirow[b]{2}{*}{$\begin{array}{c}\text { Аатентность } \\
\text { (мс) } \\
\text { Latency } \\
\text { (msec) }\end{array}$} & $\begin{array}{l}\text { Аица } \\
\text { Faces }\end{array}$ & \multirow{2}{*}{$\begin{array}{l}\text { Расстояние меж- } \\
\text { Ау координатами } \\
\text { максимальной } \\
\text { активности (мм) } \\
\text { Distance between } \\
\text { coordinates of } \\
\text { peak activity }(\mathrm{mm})\end{array}$} & $\begin{array}{c}\text { Объекты } \\
\text { Objects }\end{array}$ \\
\hline & $\begin{array}{c}\text { Аокализация фокуса } \\
\text { активности } \\
\text { Localization of the focus } \\
\text { of activity }\end{array}$ & & $\begin{array}{c}\text { Аокализация фоку- } \\
\text { са активности } \\
\text { Localization of the } \\
\text { focus of activity }\end{array}$ \\
\hline 120 & $\begin{array}{l}\text { веретенообразная из- } \\
\text { вилина (П) } \\
\text { lateral occipitotemporal } \\
\text { gyrus (R) }\end{array}$ & 97,7 & $\begin{array}{l}\text { постцентральная } \\
\text { извилина (П) } \\
\text { postcentral gyrus (R) }\end{array}$ \\
\hline 125 & $\begin{array}{l}\text { веретенообразная из- } \\
\text { вилина (А) } \\
\text { lateral occipitotemporal } \\
\text { gyrus (L) }\end{array}$ & 106,5 & $\begin{array}{l}\text { постцентральная } \\
\text { извилина (П) } \\
\text { postcentral gyrus (R) }\end{array}$ \\
\hline 130 & $\begin{array}{l}\text { постцентральная изви- } \\
\text { ^ина (П) } \\
\text { postcentral gyrus (R) }\end{array}$ & 15,8 & $\begin{array}{l}\text { постцентральная } \\
\text { извилина (П) } \\
\text { postcentral gyrus (R) }\end{array}$ \\
\hline 135 & $\begin{array}{l}\text { постцентральная изви- } \\
\text { ^ина (П) } \\
\text { postcentral gyrus (R) }\end{array}$ & 11,2 & $\begin{array}{l}\text { постцентральная } \\
\text { извилина (П) } \\
\text { postcentral gyrus (R) }\end{array}$ \\
\hline 140 & $\begin{array}{l}\text { постцентральная изви- } \\
\text { ^ина (П) } \\
\text { postcentral gyrus (R) }\end{array}$ & 15,8 & $\begin{array}{l}\text { постцентральная } \\
\text { извилина (П) } \\
\text { postcentral gyrus (R) }\end{array}$ \\
\hline
\end{tabular}




\begin{tabular}{|c|c|c|c|}
\hline \multirow[b]{2}{*}{$\begin{array}{c}\text { Аатентность } \\
\text { (мс) } \\
\text { Latency } \\
\text { (msec) }\end{array}$} & $\begin{array}{l}\text { ヘица } \\
\text { Faces }\end{array}$ & \multirow{2}{*}{$\begin{array}{c}\text { Расстояние меж- } \\
\text { ду координатами } \\
\text { максимальной } \\
\text { активности (мм) } \\
\text { Distance between } \\
\text { coordinates of } \\
\text { peak activity }(\mathrm{mm})\end{array}$} & $\begin{array}{c}\text { Oбъекты } \\
\text { Objects }\end{array}$ \\
\hline & $\begin{array}{c}\text { Аокализация фокуса } \\
\text { активности } \\
\text { Localization of the focus } \\
\text { of activity }\end{array}$ & & $\begin{array}{c}\text { Аокализация фоку- } \\
\text { са активности } \\
\text { Localization of the } \\
\text { focus of activity }\end{array}$ \\
\hline 145 & $\begin{array}{l}\text { верхняя височная изви- } \\
\text { Аина }(\wedge) \\
\text { superior temporal gyrus (L) }\end{array}$ & 129,6 & $\begin{array}{l}\text { предклинье }(\wedge) \\
\text { precuneus }(\mathrm{L})\end{array}$ \\
\hline 150 & $\begin{array}{l}\text { постцентральная изви- } \\
\text { ^ина (^) } \\
\text { postcentral gyrus (L) }\end{array}$ & 133,2 & $\begin{array}{l}\text { верхняя височная } \\
\text { извилина (П) } \\
\text { superior temporal } \\
\text { gyrus (R) }\end{array}$ \\
\hline 155 & $\begin{array}{l}\text { орбитальная } \\
\text { извилина (^) } \\
\text { orbital gyrus (L) }\end{array}$ & 132,1 & $\begin{array}{l}\text { верхняя затылочная } \\
\text { извилина }(\wedge) \\
\text { superior occipital } \\
\text { gyrus (L) }\end{array}$ \\
\hline 160 & $\begin{array}{l}\text { нижняя височная изви- } \\
\text { Аина (^) } \\
\text { inferior temporal gyrus (L) }\end{array}$ & 125,8 & $\begin{array}{l}\text { нижняя затылочная } \\
\text { изви^ина (П) } \\
\text { inferior occipital } \\
\text { gyrus (R) }\end{array}$ \\
\hline 165 & $\begin{array}{l}\text { верхняя височная изви- } \\
\text { ^ина (^) } \\
\text { superior temporal } \\
\text { gyrus (L) }\end{array}$ & 60,2 & $\begin{array}{l}\text { верхняя височная } \\
\text { извилина (П) } \\
\text { superior temporal } \\
\text { gyrus (R) }\end{array}$ \\
\hline 170 & $\begin{array}{l}\text { верхняя височная изви- } \\
\text { ^ина (^) } \\
\text { superior temporal } \\
\text { gyrus (L) }\end{array}$ & 60,2 & $\begin{array}{l}\text { верхняя височная } \\
\text { извилина (П) } \\
\text { superior temporal } \\
\text { gyrus (R) }\end{array}$ \\
\hline
\end{tabular}


Таблица 2. Расстояние межАу фокусами активности при восприятии лиц, выражающих положительные эмоции, и при восприятии объектов, вызывающих положительные эмоции

Table 2. Distance between the foci of activity when perceiving positive facial expressions and also objects generating positive emotions

\begin{tabular}{|c|c|c|c|}
\hline \multirow[b]{2}{*}{$\begin{array}{c}\text { Аатентность (мс) } \\
\text { Latency (msec) }\end{array}$} & $\begin{array}{l}\text { Аица } \\
\text { Faces }\end{array}$ & \multirow{2}{*}{\begin{tabular}{|} 
Расстояние \\
между коорди- \\
натами макси- \\
мальной актив- \\
ности (мм) \\
Distance between \\
coordinates \\
of peak \\
activity $(\mathrm{mm})$
\end{tabular}} & $\begin{array}{l}\text { Объекты } \\
\text { Objects }\end{array}$ \\
\hline & $\begin{array}{c}\text { Аокализация фоку- } \\
\text { са активности } \\
\text { Localization of the } \\
\text { focus of activity }\end{array}$ & & $\begin{array}{l}\text { Аокализация фо- } \\
\text { куса активности } \\
\text { Localization of the } \\
\text { focus of activity }\end{array}$ \\
\hline 120 & $\begin{array}{l}\text { веретенообразная } \\
\text { извилина (П) } \\
\text { lateral } \\
\text { occipitotemporal } \\
\text { gyrus (R) }\end{array}$ & 96,1 & $\begin{array}{l}\text { верхняя теменная } \\
\text { АО^ька }(\wedge) \\
\text { superior parietal } \\
\text { lobule }(L)\end{array}$ \\
\hline 125 & $\begin{array}{l}\text { средняя височная } \\
\text { извилина (П) } \\
\text { middle temporal } \\
\text { gyrus (R) }\end{array}$ & 140,7 & $\begin{array}{l}\text { предКАинье (^) } \\
\text { precuneus (L) }\end{array}$ \\
\hline 130 & $\begin{array}{l}\text { средняя височная } \\
\text { извилина (П) } \\
\text { middle temporal } \\
\text { gyrus (R) }\end{array}$ & 140,7 & $\begin{array}{l}\text { предКАинье (^) } \\
\text { precuneus (L) }\end{array}$ \\
\hline 135 & $\begin{array}{l}\text { веретенообразная } \\
\text { извилина (^) } \\
\text { lateral } \\
\text { occipitotemporal } \\
\text { gyrus (L) }\end{array}$ & 78,4 & $\begin{array}{l}\text { преАКАинье (^) } \\
\text { precuneus (L) }\end{array}$ \\
\hline 140 & $\begin{array}{l}\text { постцентральная } \\
\text { извилина (^) } \\
\text { postcentral gyrus (L) }\end{array}$ & 49,2 & $\begin{array}{l}\text { предкАинье (^) } \\
\text { precuneus (L) }\end{array}$ \\
\hline 145 & $\begin{array}{l}\text { постцентральная } \\
\text { извилина (^) } \\
\text { postcentral gyrus (L) }\end{array}$ & 49,2 & $\begin{array}{l}\text { предк^инье (^) } \\
\text { precuneus (L) }\end{array}$ \\
\hline
\end{tabular}




\begin{tabular}{|c|c|c|c|}
\hline 150 & $\begin{array}{l}\text { веретенообразная } \\
\text { извилина (^) } \\
\text { lateral } \\
\text { occipitotemporal } \\
\text { gyrus (L) }\end{array}$ & 78,4 & $\begin{array}{l}\text { предклинье (^) } \\
\text { precuneus (L) }\end{array}$ \\
\hline 155 & $\begin{array}{l}\text { нижняя затылочная } \\
\text { извилина (^) } \\
\text { inferior occipital } \\
\text { gyrus (L) }\end{array}$ & 100,8 & $\begin{array}{l}\text { постцентральная } \\
\text { извилина (^) } \\
\text { postcentral gyrus (L) }\end{array}$ \\
\hline 160 & $\begin{array}{l}\text { срединная орон- } \\
\text { тальная извилина (^) } \\
\text { middle frontal gyrus }(\mathrm{L})\end{array}$ & 123,9 & $\begin{array}{l}\text { постцентральная } \\
\text { извилина (^) } \\
\text { postcentral gyrus (L) }\end{array}$ \\
\hline 165 & $\begin{array}{l}\text { срединная фррон- } \\
\text { тальная извилина (^) } \\
\text { middle frontal gyrus }(\mathrm{L})\end{array}$ & 124,7 & $\begin{array}{l}\text { постцентральная } \\
\text { извилина (^) } \\
\text { postcentral gyrus (L) }\end{array}$ \\
\hline 170 & $\begin{array}{l}\text { срединная оррон- } \\
\text { тальная извилина (^) } \\
\text { middle frontal gyrus }(\mathrm{L})\end{array}$ & 145,2 & $\begin{array}{l}\text { предкАинье (^) } \\
\text { precuneus (L) }\end{array}$ \\
\hline
\end{tabular}

Примечание: в обеих таблицах Л - левое полушарие, П - правое полушарие. Note: in both tables $L$ indicates left hemisphere; $R$ indicates right hemisphere.

Анализ табличных данных показывает, что фокусы максимальной активности в наибольшей степени сближаются на временном интервале 130-140 мс при использовании негативных стимулов. Но даже это сравнительно небольшое расстояние (11,2-15,8 мм) не позволяет сделать вывод о перекрытии механизмов восприятия и генерации эмоций, поскольку превышает допустимую погрешность измерения ( \pm 5 мм) при использовании данного метода.

Таким образом, полученные нами результаты не выявили пересечения процессов, связанных с восприятием и воспроизведением эмоций.

\section{Обсуждение результатов}

Целью нашего исследования был поиск общих механизмов для восприятия и отображения эмоций. Для этого было необходимо определить и сравнить динамику вызванной активности при восприятии лиц с разной эмоциональной экспрессией и объектов с разной эмоциональной валентностью. Каждая такая динамика отражает целый комплекс процессов, связанных с различными этапами обработки. И нужно было подобрать стимулы и экспериментальные задачи таким образом, чтобы динамика активности при решении этих задач заведомо отличалась. Именно поэтому мы отказались от использования в качестве эмоциогенных стимулов зрительных сцен, 
поскольку наличие в них лиц, выражающих определенные эмоции, неизбежно привело бы к пересечению ответов с реакциями на стимулы-лица. В условиях же нашего эксперимента процессы обработки были предельно разведены, поскольку при восприятии лиц и объектов задействуются разные механизмы $[28,29]$. В этих условиях пересечение сравниваемых процессов возможно лишь в том случае, если существуют механизмы, общие для восприятия и воспроизведения эмоций.

Сравнение полученных реакций на лица и объекты убеждает в том, что мы действительно смогли добиться разделения сопоставляемых процессов. Сначала, естественно, фокус активности в обоих случаях располагается в зрительной коре. При этом мы не наблюдаем разницы в параметрах волны Р100. Но затем в обработку лиц и объектов вовлекаются разные корковые области [30, 31]. В это время начинают проявляться и значимые различия сравниваемых ВП.

Но прежде чем приступать к поиску пересечения механизмов восприятия и генерации эмоций, следует ответить на вопрос: как искать такое пересечение, если в каждый момент времени активируются весьма обширные, часто далеко отстоящие друг от друга области мозга? На наш взгляд, возможным решением является предположение, что именно те процессы, которые разворачиваются в фокусе максимальной корковой активности, приоритетны для актуализации. Несомненно, что оценивая эмоциональную валентность стимула, испытуемый принимает осознанное решение. Если в принятии этих решений относительно и лиц и объектов задействуются общие механизмы, то мы должны обнаружить пересечение траекторий фокусов максимальной активности.

Еще один вопрос: на каком временном отрезке следует искать пересечение сравниваемых процессов? Литературные данные указывают на то, что операции, связанные с определением эмоциональных выражений лиц, реализуются во время формирования волны N170 [32, 33, 34]. М. Эймер и А. Холмс еще более точно указывают на интересующий нас временной интервал: со 120-й по 180-ю мс [35]. Правда, именно на этот временной диапазон приходятся наиболее выраженные из обнаруженных нами различий сравниваемых ВП.

Результаты сопоставления траекторий фокусов максимальной активности на этом временном интервале также указывают на отсутствие пересечения сравниваемых процессов.

Но правильно ли ожидать, что сама операция «отзеркаливания» при генерации эмоций должна происходить в то же самое время, в которое эти механизмы активируются при восприятии эмоций? На самом деле, это может происходить в разное время. Главное, чтобы активация затрагивала одни и те 
же структуры. Для ответа на этот вопрос мы сравнили полные траектории фокусов активности при реализации сравниваемых процессов. Мы искали любые их пересечения, даже если одни и те же участки коры активируются лицами и объектами в разное время. Однако не обнаружили их ни в левом, ни в правом полушарии.

Конечно, было бы неправильно утверждать, что мы, наконец, получили ответ на вопрос, который дебатируется уже десятки лет. Но обнаруженные нами новые факты позволяют сделать очередной шаг в решении проблемы функциональной эквивалентности в эмоциональной сфере. Это стало возможным благодаря двум принципиальным методическим приемам: мы разделили задачи восприятия и генерации эмоций не только во времени, но и с помощью «непересекающихся» стимулов; мы сравнили реакции, полученные на одних и тех же испытуемых в ходе одного эксперимента.

\section{Заключение}

Анализ и сопоставление динамики активности мозговых структур не выявили пересечения процессов, реализуемых в задаче распознавания эмоционального выражения лиц и в задаче определения эмоциональной валентности объектов. Это может указывать на отсутствие механизмов, общих для восприятия и генерации эмоций.

\section{Литература}

1. Shepard R. N., Metzler J. Mental rotation of three-dimensional objects // Science. - 1971. - Vol. 171, № 3972. - P. 701-703.

2. Kosslyn S. M., Ball T. M., Reiser B. J. Visual images preserve metric spatial information: evidence from studies of image scanning // Journal of experimental psychology: Human perception and performance. - 1978. - Vol. 4, № 1. - P. 47-60.

3. Holmes P. S., Cumming J., Edwards M. G. Motor imagery in learning processes: Motor imagery and observation in skill learning / Guillot A., Collet C. (eds.) The neurophysiological foundations of mental and motor imagery. - Oxford, UK : Oxford University Press, 2010. - P. 253-269.

4. Moran A., Guillot A., Macintyre T., Collet C. Re-imagining motor imagery: Building bridges between cognitive neuroscience and sport psychology // British Journal of Psychology. - 2012. - Vol. 103, № 2. - P. 224-247. - DOI: 10.1111/j.2044-8295.2011.02068.x

5. Bastiaansen J. A. C. J., Thioux M., Keysers C. Evidence for mirror systems in emotions // Philosophical Transactions of the Royal Society of London B: Biological Sciences. - 2009. - Vol. 364, № 1528. - P. 2391-2404. - DOI: 10.1098/ rstb.2009.0058 
6. Lamm C., Bukowski H., Silani G. From shared to distinct self-other representations in empathy: evidence from neurotypical function and socio-cognitive disorders // Philosophical Transactions of the Royal Society B. - 2016. Vol. 371, Issue 1686. - 20150083. - DOI: 10.1098/rstb.2015.0083

7. Decety J., Grèzes J. The power of simulation: imagining one's own and other's behavior // Brain Research. - 2006. - Vol. 1079, № 1. - P. 4-14. - DOI: 10.1016/j.brainres.2005.12.115

8. Leslie K. R., Johnson-Frey S. H., Grafton S. T. Functional imaging of face and hand imitation: towards a motor theory of empathy // Neuroimage. - 2004. Vol. 21, № 2. - P. 601-607. - DOI: 10.1016/j.neuroimage.2003.09.038

9. Singer T., Lamm C. The social neuroscience of empathy // Annals of the New York Academy of Sciences. - 2009. - Vol. 1156, № 1. - P. 81-96. DOI: $10.1111 /$ j.1749-6632.2009.04418.x

10. Decety J. To what extent is the experience of empathy mediated by shared neural circuits? // Emotion Review. - 2010. - Vol. 2, № 3. - P. 204-207. - DOI: $10.1177 / 1754073910361981$

11. Rütgen M., Seidel E.-M., Silani G., Riecansky I., Hummer A., Windischberger C., Petrovic P., Lamm C. Placebo analgesia and its opioidergic regulation suggest that empathy for pain is grounded in self pain // Proceedings of the National Academy of Sciences. - 2015. - Vol. 112, № 41. - P. E5638-E5646. DOI: $10.1073 /$ pnas.1511269112

12. Han X., He K., Wu B., Shi Z., Liu Y., Luo S., Wei K., Wu X., Han S. Empathy for pain motivates actions without altruistic effects: evidence of motor dynamics and brain activity // Social Cognitive and Affective Neuroscience. - 2017. Vol. 12, № 6. - P. 893-901. - DOI: 10.1093/scan/nsx016

13. Carr L., lacoboni M., Dubeau M.-C., Mazziotta J. C., Lenzi G. L. Neural mechanisms of empathy in humans: a relay from neural systems for imitation to limbic areas // Proceedings of the national Academy of Sciences. - 2003. - Vol. 100, № 9. - P. 5497-5502. - DOI: 10.1073/pnas.0935845100

14. Wicker B., Keyser C., Plailly J., Royet J. P., Gallese V., Rizzolatti G. Both of us disgusted in my insula: the common neural basis of seeing and feeling disgust // Neuron. 2003. - Vol. 40, № 3. - P. 655-664. - DOI: 10.1016/S0896-6273(03)00679-2

15. Heller A. S., Lapate R. C., Mayer K. E., Davidson R. J. The face of negative affect: trial-by-trial corrugator responses to negative pictures are positively associated with amygdala and negatively associated with ventromedial prefrontal cortex activity // Journal of Cognitive Neuroscience. - 2014. Vol. 26, № 9. - P. 2102-2110. - DOI: 10.1162/jocn_a_00622

16. Lamm C., Majdandžić J. The role of shared neural activations, mirror neurons, and morality in empathy - A critical comment // Neuroscience Research. 2015. - Vol. 90. - P. 15-24. - DOI: 10.1016/j.neures.2014.10.008 
17. Britton J. C., Britton C., Taylor S. F., Sudheimer K. D., Liberzon I. Facial expressions and complex IAPS pictures: common and differential networks // Neuroimage. - 2006. - Vol. 31, № 2. - P. 906-919. - DOI: 10.1016/j.neuroimage.2005.12.050

18. Olszanowski M., Pochwatko G., Kuklinski K., Scibor-Rylski M., Lewinski P., Ohme R. K. Warsaw set of emotional facial expression pictures: a validation study of facial display photographs // Frontiers in psychology. - 2014. Vol. 5. - 1516. - DOI: 10.3389/fpsyg.2014.01516

19. Pantic M., Valstar M., Rademaker R., Maat L. Web-based database for facial expression analysis // IEEE International Conference on Multimedia and Expo (ICME, 2005). - DOI: 10.1109/ICME.2005.1521424

20. Lundqvist D., Flykt A., Öhman A. The Karolinska directed emotional faces (KDEF). Stockholm, Sweden : Karolinska Institute, Department of Clinical Neuroscience, Psychology Section, 1998.

21. Delorme A., Makeig S. EEGLAB: an open source toolbox for analysis of singletrial EEG dynamics // Journal of Neuroscience Methods. - 2004. - Vol. 134, № 1. - P. 9-21. - DOI: 10.1016/j.jneumeth.2003.10.009

22. Pascual-Marqui R. D. Standardized low-resolution brain electromagnetic tomography (sLORETA): Technical details // Methods and Findings in Experimental and Clinical Pharmacology. - 2002. - Vol. 24. - P. 5-12.

23. Maratos F. A., Garner M., Karl A., Hogan A. M. When is a face a face? Schematic faces, emotion, attention and the N170 // AIMS Neuroscience. - 2015. - Vol. 2, № 3. - P. 172-182. - DOI: 10.3934/Neuroscience.2015.3.172

24. Almeida P. R., Ferreira-Santos F., Chaves P. L., Paiva T. O., Barbosa F., MarquesTeixeira J. Perceived arousal of facial expressions of emotion modulates the N170, regardless of emotional category: Time domain and time-frequency dynamics // International Journal of Psychophysiology. - 2016. - Vol. 99. P. 48-56. - DOI: 10.1016/j.ijpsycho.2015.11.017

25. Almeida P. R., Ferreira-Santos F., Vieira J. B., Moreira P. S., Barbosa F., MarquesTeixeira J. Dissociable effects of psychopathic traits on cortical and subcortical visual pathways during facial emotion processing: an ERP study on the N170 // Psychophysiology.-2014. - Vol. 51, № 7.-P.645-657.-DOI: 10.1111/psyp.12209

26. Lin H., Schulz C., Straube T. Contextual effects of surprised expressions on the encoding and recognition of emotional target faces: An event-related potential (ERP) study // Biological Psychology. - 2017. - Vol. 129. - P. 273-281. DOI: 10.1016/j.biopsycho.2017.09.011

27. Cacioppo J. T., Tassinary L. G., Berntson G. Handbook of Psychophysiology. 3rd Edition. - Cambridge : Cambridge University Press, 2007. - 908 p.

28. Rice G. E., Watson D. M., Hartley T., Andrews T. J. Low-Level Image Properties of Visual Objects Predict Patterns of Neural Response across Category-Selective 
Regions of the Ventral Visual Pathway // Journal of Neuroscience. - 2014. Vol. 34, № 26. - P. 8837-8844. - DOI: 10.1523/JNEUROSCI.5265-13.2014

29. Zhen Z., Yang Z., Huang L., Kong X.-Z., Wang X., Dang X., Huang Y., Song Y., Liu J. Quantifying interindividual variability and asymmetry of face-selective regions: A probabilistic functional atlas // Neurolmage. - 2015. - Vol. 113. P. 13-25. - DOI: 10.1016/j.neuroimage.2015.03.010

30. Goffaux V., Peters J., Haubrechts J., Schiltz C., Jansma B., Goebel R. From coarse to fine? Spatial and temporal dynamics of cortical face processing // Cerebral Cortex. - 2010. - Vol. 21, № 2. - P. 467-476. - DOI: 10.1093/cercor/bhq112

31. Grill-Spector K., Weiner K. S. The functional architecture of the ventral temporal cortex and its role in categorization // Nature Reviews Neuroscience. 2014. - Vol. 15, № 8. - P. 536-548. - DOI: 10.1038/nrn3747

32. Hietanen J. K., Astikainen P. N170 response to facial expressions is modulated by the affective congruency between the emotional expression and preceding affective picture // Biological psychology. - 2013. - Vol. 92, № 2. - P. 114-124. - DOI: 10.1016/j.biopsycho.2012.10.005

33. Hinojosa J. A., Mercado F., Carretié L. N170 sensitivity to facial expression: a meta-analysis // Neuroscience \& Biobehavioral Reviews. - 2015. - Vol. 55. P. 498-509. - DOI: 10.1016/j.neubiorev.2015.06.002

34. Wang X., Jin J., Liu Z., Yin T. Study on Differences of Early-Mid ERPs Induced by Emotional Face and Scene Images // International Symposium on Neural Networks. - Springer, Cham, 2017.- P.550-558. - DOI: 10.1007/978-3-319-59081-3

35. Eimer M., Holmes A. Event-related brain potential correlates of emotional face processing // Neuropsychologia. - 2007. - Vol. 45, № 1. - P. 15-31. DOI: 10.1016/j.neuropsychologia.2006.04.022

\section{References}

1. Shepard R. N., Metzler J. Mental rotation of three-dimensional objects. Science, 1971, V. 171, no. 3972, pp. 701-703.

2. Kosslyn S. M., Ball T. M., Reiser B. J. Visual images preserve metric spatial information: evidence from studies of image scanning. Journal of experimental psychology: Human perception and performance, 1978, V. 4, no. 1, pp. 47-60.

3. Holmes P. S., Cumming J., Edwards M. G. Motor imagery in learning processes: Motor imagery and observation in skill learning. In: Guillot A., Collet C. (eds.) The neurophysiological foundations of mental and motor imagery. Oxford, UK, Oxford University Press, 2010, pp. 253-269.

4. Moran A., Guillot A., Macintyre T., Collet C. Re-imagining motor imagery: Building bridges between cognitive neuroscience and sport psychology. British Journal of Psychology, 2012, V. 103, no. 2, pp. 224-247. DOI: 10.1111/j.2044-8295.2011.02068.x 
5. Bastiaansen J. A. C. J., Thioux M., Keysers C. Evidence for mirror systems in emotions. Philosophical Transactions of the Royal Society of London B: Biological Sciences, 2009, V. 364, no. 1528, pp. 2391-2404. DOI: 10.1098/rstb.2009.0058

6. Lamm C., Bukowski H., Silani G. From shared to distinct self-other representations in empathy: evidence from neurotypical function and socio-cognitive disorders. Philosophical Transactions of the Royal Society B, 2016, V. 371, Issue 1686, 20150083. DOI: 10.1098/rstb.2015.0083

7. Decety J., Grèzes J. The power of simulation: imagining one's own and other's behavior. Brain Research, 2006, V. 1079, no. 1, pp. 4-14. DOI: 10.1016/j. brainres.2005.12.115

8. Leslie K. R., Johnson-Frey S. H., Grafton S. T. Functional imaging of face and hand imitation: towards a motor theory of empathy. Neuroimage, 2004, V. 21, no. 2, pp. 601-607. DOI: 10.1016/j.neuroimage.2003.09.038

9. Singer T., Lamm C. The social neuroscience of empathy. Annals of the New York Academy of Sciences, 2009, V. 1156, no. 1, pp. 81-96. DOI: 10.1111/j.17496632.2009.04418.x

10. Decety J. To what extent is the experience of empathy mediated by shared neural circuits? Emotion Review, 2010, V. 2, no. 3, pp. 204-207. DOI: 10.1177/1754073910361981

11. Rütgen M., Seidel E.-M., Silani G., Riecansky I., Hummer A., Windischberger C., Petrovic P., Lamm C. Placebo analgesia and its opioidergic regulation suggest that empathy for pain is grounded in self pain. Proceedings of the National Academy of Sciences, 2015, V. 112, no. 41, pp. E5638-E5646. DOI: 10.1073/ pnas.1511269112

12. Han X., He K., Wu B., Shi Z., Liu Y., Luo S., Wei K., Wu X., Han S. Empathy for pain motivates actions without altruistic effects: evidence of motor dynamics and brain activity. Social Cognitive and Affective Neuroscience, 2017, V. 12, no. 6, pp. 893-901. DOI: 10.1093/scan/nsx016

13. Carr L., lacoboni M., Dubeau M.-C., Mazziotta J. C., Lenzi G. L. Neural mechanisms of empathy in humans: a relay from neural systems for imitation to limbic areas. Proceedings of the national Academy of Sciences, 2003, V. 100, no. 9, pp. 5497-5502. DOI: 10.1073/pnas.0935845100

14. Wicker B., Keyser C., Plailly J., Royet J. P., Gallese V., Rizzolatti G. Both of us disgusted in my insula: the common neural basis of seeing and feeling disgust. Neuron, 2003, V. 40, no. 3, pp. 655-664. DOI: 10.1016/S0896-6273(03)00679-2

15. Heller A. S., Lapate R. C., Mayer K. E., Davidson R. J. The face of negative affect: trial-by-trial corrugator responses to negative pictures are positively associated with amygdala and negatively associated with ventromedial prefrontal cortex activity. Journal of Cognitive Neuroscience, 2014, V. 26, no. 9, pp. 2102-2110. DOI: 10.1162/jocn_a_00622 
16. Lamm C., Majdandžić J. The role of shared neural activations, mirror neurons, and morality in empathy - A critical comment. Neuroscience Research, 2015, V. 90, pp. 15-24. DOI: 10.1016/j.neures.2014.10.008

17. Britton J. C., Britton C., Taylor S. F., Sudheimer K. D., Liberzon I. Facial expressions and complex IAPS pictures: common and differential networks. Neuroimage, 2006, V. 31, no. 2, pp. 906-919. DOI: 10.1016/j.neuroimage.2005.12.050

18. Olszanowski M., Pochwatko G., Kuklinski K., Scibor-Rylski M., Lewinski P., Ohme R. K. Warsaw set of emotional facial expression pictures: a validation study of facial display photographs. Frontiers in psychology. 2014, V. 5, 1516. DOI: $10.3389 /$ fpsyg.2014.01516

19. Pantic M., Valstar M., Rademaker R., Maat L. Web-based database for facial expression analysis. IEEE International Conference on Multimedia and Expo (ICME, 2005). DOI: 10.1109/ICME.2005.1521424

20. Lundqvist D., Flykt A., Öhman A. The Karolinska directed emotional faces (KDEF). Stockholm, Sweden, Karolinska Institute, Department of Clinical Neuroscience, Psychology Section, 1998.

21. Delorme A., Makeig S. EEGLAB: an open source toolbox for analysis of single-trial EEG dynamics. Journal of Neuroscience Methods, 2004, V. 134, no. 1, pp. 9-21. DOI: 10.1016/j.jneumeth.2003.10.009

22. Pascual-Marqui R. D. Standardized low-resolution brain electromagnetic tomography (sLORETA): Technical details. Methods and Findings in Experimental and Clinical Pharmacology, 2002, V. 24, pp. 5-12.

23. Maratos F. A., Garner M., Karl A., Hogan A. M. When is a face a face? Schematic faces, emotion, attention and the N170. AIMS Neuroscience, 2015, V. 2, no. 3, pp. 172-182. DOI: 10.3934/Neuroscience.2015.3.172

24. Almeida P. R., Ferreira-Santos F., Chaves P. L., Paiva T. O., Barbosa F., MarquesTeixeira J. Perceived arousal of facial expressions of emotion modulates the N170, regardless of emotional category: Time domain and time-frequency dynamics. International Journal of Psychophysiology, 2016, V. 99, pp. 48-56. DOI: 10.1016/j.ijpsycho.2015.11.017

25. Almeida P. R., Ferreira-Santos F., Vieira J. B., Moreira P. S., Barbosa F., MarquesTeixeira J. Dissociable effects of psychopathic traits on cortical and subcortical visual pathways during facial emotion processing: an ERP study on the N170. Psychophysiology, 2014, V. 51, no. 7, pp. 645-657. DOI: 10.1111/psyp.12209

26. Lin H., Schulz C., Straube T. Contextual effects of surprised expressions on the encoding and recognition of emotional target faces: An event-related potential (ERP) study. Biological Psychology, 2017, V. 129, pp. 273-281. DOI: 10.1016/j.biopsycho.2017.09.011

27. Cacioppo J. T., Tassinary L. G., Berntson G. Handbook of psychophysiology. 3rd Edition. Cambridge, Cambridge University Press, 2007. 908 p. 
28. Rice G. E., Watson D. M., Hartley T., Andrews T. J. Low-level image properties of visual objects predict patterns of neural response across category-selective regions of the ventral visual pathway. Journal of Neuroscience, 2014, V. 34, no. 26, pp. 8837-8844. DOI: 10.1523/JNEUROSCI.5265-13.2014

29. Zhen Z., Yang Z., Huang L., Kong X.-Z., Wang X., Dang X., Huang Y., Song Y., Liu J. Quantifying interindividual variability and asymmetry of face-selective regions: A probabilistic functional atlas. Neurolmage, 2015, V. 113, pp. 13-25. DOI: 10.1016/j.neuroimage.2015.03.010

30. Goffaux V., Peters J., Haubrechts J., Schiltz C., Jansma B., Goebel R. From coarse to fine? Spatial and temporal dynamics of cortical face processing. Cerebral Cortex, 2010, V. 21, no. 2, pp. 467-476. DOI: 10.1093/cercor/bhq112

31. Grill-Spector K., Weiner K. S. The functional architecture of the ventral temporal cortex and its role in categorization. Nature Reviews Neuroscience, 2014, V. 15, no. 8, pp. 536-548. DOI: 10.1038/nrn3747

32. Hietanen J. K., Astikainen P. N170 response to facial expressions is modulated by the affective congruency between the emotional expression and preceding affective picture. Biological Psychology, 2013, V. 92, no. 2, pp. 114-124. DOI: 10.1016/j.biopsycho.2012.10.005

33. Hinojosa J. A., Mercado F., Carretié L. N170 sensitivity to facial expression: a meta-analysis. Neuroscience \& Biobehavioral Reviews, 2015, V. 55, pp. 498-509. DOI: 10.1016/j.neubiorev.2015.06.002

34. Wang X., Jin J., Liu Z., Yin T. Study on differences of early-mid ERPs induced by emotional face and scene images. International Symposium on Neural Networks. Springer, Cham, 2017, pp. 550-558. DOI: 10.1007/978-3-319-59081-3

35. Eimer M., Holmes A. Event-related brain potential correlates of emotional face processing. Neuropsychologia, 2007, V. 45, no. 1, pp. 15-31. DOI: 10.1016/j. neuropsychologia.2006.04.022 